\title{
Increasing user confidence in intelligent environments
}

\author{
Miguel J. Hornos ${ }^{1}$ · Carlos Rodríguez-Domínguez ${ }^{1}$
}

Published online: 25 May 2018

(c) Springer International Publishing AG, part of Springer Nature 2018

\section{Introduction}

The title Increasing User Confidence in Intelligent Environments of this special issue, which gathers a selection of papers comprising different aspects of the development of safer, securer, and more reliable Intelligent Environments, tries to emphasize the fact that user confidence must be a crucial facet to be taken into account by all those involved in the research and development of this type of systems. In fact, from the very beginning of Intelligent Environments (IE) [1], and related disciplines, such as Ambient Intelligence (AmI) [2] and Smart Environments (SmE) [3], a strong stress has been put on the users, to whom the corresponding system (i.e., the IE) must assist, help, and serve, simply because, without them, it would lose its raison d'être.

This special issue aims to expand in more detail some of the most interesting and inspiring issues addressed in the 6th International Workshop on the Reliability of Intelligent Environments (WoRIE 2017) ${ }^{1}$, while strengthening and making more visible the close relationship between the workshop and this journal. Nonetheless, an open call for papers allowed other researchers from outside the workshop to submit contributions to this special issue. In this way, we intended to have a greater number of submissions, in addition to contributing to increase the sense of community among the researchers and practitioners involved in the field of the development of reliable IE. This last objective is shared with WoRIE, which tries to be a forum to build firm bridges of collaboration among all the professionals belonging to the different disciplines and

\footnotetext{
1 http://www.ugr.es/ worie/2017/.

$凶$ Miguel J. Hornos

mhornos@ugr.es

Carlos Rodríguez-Domínguez

carlosrodriguez@ugr.es

1 MYDASS (Modelling and Development of Advanced Software Systems) Research Group, Software Engineering Department, Higher Technical School of Computer and Telecommunication Engineering, Campus de Aynadamar, University of Granada, 18071 Granada, Spain
}

groups involved in the research and development of more reliable, safer, and securer IE.

The importance of IE comes from they are systems that can face several societal challenges, like the ageing of the population in most developed societies or the need of a more sustainable economy model. Accordingly, the development of IE has become a popular research field that aims for the transformation of our lifestyle at different levels and domains: healthcare, social inclusion, people assistance, quality of life, labor productivity, lifelong learning, entertainment, and intelligent and adapted transport, among others.

To do that, IE involve a large-scale deployment of sensor/actuator networks and the use of a middleware, communication protocols, Artificial Intelligence techniques, location/positioning methods, smart applications, and other sophisticate technologies [1-3]. IE are also context-aware systems [4] that need to identify the specific situations and users' preferences [5] to provide custom services adapted to the users' needs. To achieve this goal, human activity recognition is also necessary, e.g., using wearable sensors [6]. Hence, the scientific field of IE is a multidisciplinary domain [1], which embraces researchers and practitioners of diverse technical disciplines, such as Software Engineering, Ubiquitous/Pervasive Computing, Artificial Intelligence, Robotics and Human-Computer Interaction, among others. These interdisciplinary professionals are collaborating to develop increasingly complex, yet more reliable and effective, systems to really improve human lifestyle through the provision of solutions to the above-mentioned challenges.

As it can be easily inferred, reliability assessment is a strong requirement in IE, since there might be different sources of failures in them, such as: sensor measurements, connection disruptions, unexpected human behaviour, etc. Without going further, a self-driving car (which can be classified as an IE) did not stop when a woman crossed the road and killed her last March [7]. This example clearly shows that the development of these intricate systems is a hard and challenging task, where reliability, safety, and security should be central and critical aspects to be guaranteed. Therefore, additional efforts are required to design systems in which people can trust [8]. 
Consequently, the application of formal and/or semiformal methods and techniques, such as the ones coming from Software Engineering [9] (e.g., specification, modelling, verification, validation, simulation, testing, etc.), is needed to analyze and establish the correctness of IE, which are complex and error-prone systems, with the aim of improving their reliability, safety, and/or security, and, therefore, increasing the user confidence in these systems.

So far, some methodologies have been specifically proposed to improve the reliability of IE [10-12]. Likewise, other methodologies initially proposed for the development of reliable Pervasive/Ubiquitous Computing [13-15], Ambient Assisted Living (AAL) [16,17], and Multi-Agent [18] Systems could be applied to the development of IE. However, these proposals still face two main challenges, yet to be solved: (1) such methodologies are usually intended to be applied only in some of the development stages; (2) there is a lack of integration between them [4]. In consequence, greater efforts are necessary to provide more integrated and holistic methodologies, which should include the corresponding techniques and tools, so that they can be commonly adopted and used by the professionals involved in the development of this type of systems.

Keeping all this in mind, this special issue aims to compile recent advances to increase the user confidence in IE, while showing convincing examples and the application of best practices in diverse germane domains.

\section{Contents of this special issue}

This section contains a synopsis of the three articles that have been selected to be published in this special issue. We intend that this serves as a kind of brief presentation of their contents that makes the reader interested in the topics covered in them and encourages him/her to read them.

In the first paper [19], Moriyama et al. propose a communication control system that uses acoustic waves to share information between portable devices in a securer way than using the traditional communication methods based on radio waves, such as Wi-Fi or Bluetooth. Unlike the latter, in their proposal, the intensity and frequency of the output acoustic signal emitted by the sender can be adjusted to respectively control the distance and angle where the receiver is. In this way, this receiver device can be distinguished from other devices which are in the same room. Hence, breaching the communication between two devices is considerably more difficult using this control mechanism, since it would only be possible to do so if the eavesdropping device is placed right in their communication path, while using the existing radio-based approaches, it would be possible to do it in any direction and even beyond walls. Consequently, security is improved with respect to such methods.
In the second paper [20], Suh, Lee, and Cho present a software framework, named ICARS (Integrated Control Architecture for Robotic mediator in Smart environments), to enable the integration of mediator robots into ambient intelligence environments. The main goal is to improve the reliability of the whole system while integrating mediator robots in it, which is a complex yet very important task to increase user acceptance of these novel technologies that will provide user assistance. An overview of publications in that research field is provided too. In comparison to them, the authors introduce some interesting extensions to the different stages involved in the development of an intelligent environment. Finally, a preliminary validation of the framework is also presented, showing some encouraging results that should be taken into consideration by other future contributions. Particularly, this research can be a promising approach to address user concerns related to the use of mediator robots as personal assistants in smart environments.

Last but not least, in the third paper [21], Given-Wilson, Legay, Sedwards, and Zendra propose the ACANTO project to develop robotic assistants helping older adults engaged in therapeutic activities to navigate through complex physical environments. A key contribution of ACANTO is that it addresses navigation in multi-user, multi-robot environments. In comparison, most of the current scientific literature in this field is oriented towards solving this open matter in single-user, single-robot environments. The presented solution involves a motion planner that is able to readapt the navigation plan in real time using algorithms based on the Social Force Model and Statistical Model Checking. A trace and group inference mechanism is able to predict the behaviour of a set of users at the same time. An experimental validation shows the feasibility of this proposal, particularly focusing on showing its effectiveness and efficiency.

\section{References}

1. Augusto JC, Callaghan V, Cook D, Kameas A, Satoh I (2013) Intelligent Environments: a manifesto. Human-centric. Comput Inf Sci 3:12. https://doi.org/10.1186/2192-1962-3-12

2. Ramos C, Augusto JC, Shapiro D (2008) Ambient Intelligencethe Next Step for Artificial Intelligence. IEEE Intell Syst 23(2):1518

3. Cook D, Das SK (2005) Smart environments: technology, protocols and applications. Wiley, Hoboken

4. Alegre U, Augusto JC, Clark T (2016) Engineering context-aware systems and applications: A survey. J Syst Softw 117:55-83

5. Oguego CL, Augusto JC, Muñoz A, Springett M (2018) A survey on managing users' preferences in ambient intelligence. Univ Access Inf Soc 17(1):97-114

6. Lara OD, Labrador MA (2013) A survey on human activity recognition using wearable sensors. IEEE Commun Surv Tutor 15(3):1192-1209

7. The Guardian (2018) Video released of Uber self-driving crash that killed woman in Arizona. https://www.theguardian.com/ 
technology/2018/mar/22/video-released-of-uber-self-drivingcrash-that-killed-woman-in-arizona

8. Corno F, Guercio E, De Russis L, Gargiulo E (2015) Designing for user confidence in intelligent environments. J Reliab Intell Environ $1(1): 11-21$

9. Hornos MJ (2017) Application of Software Engineering techniques to improve the reliability of Intelligent Environments. J Reliab Intell Environ 3(1):1-3

10. Augusto JC, Hornos MJ (2013) Software simulation and verification to increase the reliability of Intelligent Environments. Adv Eng Softw 58:18-34

11. Preuveneers D, Joosen W (2016) Semantic analysis and verification of context-driven adaptive applications in intelligent environments. J Reliab Intell Environ 2(2):53-73

12. Le Guilly T, Nielsen MK, Pedersen T, Skou A, Kjeldskov J, Skov M (2016) User constraints for reliable user-defined smart home scenarios. J Reliab Intell Environ 2(2):75-91

13. Coronato A, De Pietro G (2011) Formal specification and verification of ubiquitous and pervasive systems. ACM Trans Auton Adapt Syst 6(1):9. https://doi.org/10.1145/1921641.1921650

14. Hallsteinsen S, Geihs K, Paspallis N, Eliassen F, Horn G, Lorenzo J, Mamelli A, Papadopoulos GA (2012) A development framework and methodology for self-adapting applications in ubiquitous computing environments. J Syst Softw 85(12):2840-2859
15. Tang L, Yu Z, Wang H, Zhou X, Duan Z (2014) Methodology and tools for pervasive application development. Int J Distrib Sensor Netw. https://doi.org/10.1155/2014/516432 (Article ID 516432)

16. Benghazi K, Hurtado MV, Hornos MJ, Rodríguez ML, RodríguezDomínguez C, Pelegrina AB, Rodríguez-Fórtiz MJ (2012) Enabling correct design and formal analysis of Ambient Assisted Living systems. J Syst Softw 85(3):498-510

17. Coronato A, Paragliola G (2017) A structured approach for the designing of safe AAL applications. Expert Syst Appl 85:1-13

18. Augusto JC, Hornos MJ (2012) Designing More Reliable MASbased Ambient Intelligence Systems. In: Bosse T (ed) Agents and Ambient Intelligence. Ambient Intelligence and Smart Environments, vol. 12. IOS Press, Amsterdam, pp 65-90

19. Moriyama S, Kawakita Y, Takahashi J, Tobe Y (2018) Controlling of communication connection range using acoustic waves emitted from smartphones. J Reliab Intell Environ. https://doi.org/10.1007/ s40860-018-0059-0

20. Suh YH, Lee KW, Cho ES (2018) A software framework for robotic mediators in smart environments. Journal of Reliable Intelligent Environments. https://doi.org/10.1007/s40860-018-0060-7

21. Given-Wilson T, Legay A, Sedwards S, Zendra O (2018) Group abstraction for assisted navigation of social activities in intelligent environments. J Reliab Intell Environ. https://doi.org/10. 1007/s40860-018-0058-1 